\title{
Comparative genomic and functional analysis of Akkermansia muciniphila and closely related species
}

\author{
Juyuan Xing ${ }^{2} \cdot{\text { Xiaobo } \mathrm{Li}^{3,5,6} \cdot \text { Yingjiao Sun }^{1} \cdot \text { Juanjuan Zhao }}^{1} \cdot$ Shaohua Miao ${ }^{1}$ Q Qin Xiong ${ }^{1} \cdot$ Yonggang Zhang $^{4}$. \\ Guishan Zhang'
}

Received: 13 March 2019 / Accepted: 18 July 2019 / Published online: 9 August 2019

(c) The Author(s) 2019

\begin{abstract}
Background Akkermansia muciniphila is an important bacterium that resides on the mucus layer of the intestinal tract. Akkermansia muciniphila has a high abundance in human feces and plays an important role in human health.

Objective In this article, 23 whole genome sequences of the Akkermansia genus were comparatively studied.

Methods Phylogenetic trees were constructed with three methods: All amino acid sequences of each strain were used to construct the first phylogenetic tree using the web server of Composition Vector Tree Version 3. The matrix of Genome-toGenome Distances which were obtained from GGDC 2.0 was used to construct the second phylogenetic tree using FastME. The concatenated single-copy core gene-based phylogenetic tree was generated through MEGA. The single-copy genes were obtained using OrthoMCL. Population structure was assessed by STRUCTURE 2.3.4 using the SNPs in core genes. PROKKA and Roary were used to do pan-genome analyses. The biosynthetic gene clusters were predicted using antiSMASH 4.0. IalandViewer 4 was used to detect the genomic islands.

Results The results of comparative genomic analysis revealed that: (1) The 23 Akkermansia strains formed 4 clades in phylogenetic trees. The A. muciniphila strains isolated from different geographic regions and ecological niches, formed a closely related clade. (2) The 23 Akkermansia strains were divided into 4 species based on digital DNA-DNA hybridization (dDDH) values. (3) Pan-genome of A. muciniphila is in an open state and increases with addition of new sequenced genomes. (4) SNPs were not evenly distributed throughout the A. muciniphila genomes. The genes in regions with high SNP density are related to metabolism and cell wall/membrane envelope biogenesis. (5) The thermostable outer-membrane protein, Amuc_1100, was conserved in the Akkermansia genus, except for Akkermansia glycaniphila $\mathrm{Pyt}^{\mathrm{T}}$.

Conclusion Overall, applying comparative genomic and pan-genomic analyses, we classified and illuminated the phylogenetic relationship of the 23 Akkermansia strains. Insights of the evolutionary, population structure, gene clusters and genome islands of Akkermansia provided more information about the possible physiological and probiotic mechanisms of the Akkermansia strains, and gave some instructions for the in-depth researches about the use of Akkermansia as a gut probiotic in the future.
\end{abstract}

Keywords Akkermansia muciniphila $\cdot$ Comparative genome $\cdot$ Phylogenetic analysis $\cdot$ Pan-genome $\cdot$ Gene clusters

Juyuan Xing and Xiaobo Li co-first author and equal contribution.

Electronic supplementary material The online version of this article (https://doi.org/10.1007/s13258-019-00855-1) contains supplementary material, which is available to authorized users.

Yonggang Zhang

zhangygcq@163.com

Guishan Zhang gszhang86@gmail.com

Extended author information available on the last page of the article

\section{Introduction}

Akkermansia muciniphila, a new member of the Verrucomicrobia phylum (Hedlund et al. 1997), was first isolated from human feces as a new mucus-degrading bacterium in 2004 (Derrien et al. 2004). A. muciniphila, which is an anaerobic Gram-negative bacterium commonly found within the mucus layer of the digestive tract, is isolated by anaerobic medium containing gastric mucin as the sole carbon and nitrogen source (Derrien et al. 2004). Thus far, there are two species in the Akkermansia genus as follows: A. muciniphila 
ATCC BAA $-835^{\mathrm{T}}$, which was first isolated from human feces in 2004 (Derrien et al. 2004); and Akkermansia glycaniphila $\mathrm{Pyt}^{\mathrm{T}}$, which was first isolated from reticulated python feces in 2016 (Ouwerkerk et al. 2016). Metagenome data suggest that there are at least eight different species of the Akkermansia genus in the digestive tract of humans apart from $A$. muciniphila, and different species exist in the same region simultaneously (van Passel et al. 2011). The differences of ecological niches and hosts of Akkermansia are tremendous. Akkermansia-like organisms are found in the intestines of other non-human mammals, such as lemur, gorilla (Ley et al. 2008) and mice (Presley et al. 2010), as well as other vertebrates, such as chickens (Belzer and de Vos 2012) and zebrafish (Roeselers et al. 2011). Moreover, 16S rRNA sequences of the Akkermansia genus are universally detected in animals, ranging from domesticated and wild mammals to non-mammals, such as birds and fish (Belzer and de Vos 2012), as well as to reptiles, such as the Burmese python (Costello et al. 2010). The gastrointestinal tract anatomy, diet and mucin types of these animals are largely different.

The first available A. muciniphila genome sequence was for the strain ATCC BAA-835 ${ }^{\mathrm{T}}$, which was sequenced in 2011 (van Passel et al. 2011). The whole genome of this strain is composed of one circular chromosome of $2.66 \mathrm{Mbp}$ with an average $\mathrm{G}+\mathrm{C}$ content of $55.8 \%$. The A. glycaniphila Pyt $^{\mathrm{T}}$ genome was sequenced in 2017 (Ouwerkerk et al. 2017), comprising one circular chromosome of 3.07 $\mathrm{Mbp}$ with a $\mathrm{G}+\mathrm{C}$ content of $57.6 \%$. Compared with other genomes of the Verrucomicrobia phylum, the A. muciniphila genome showed distinct phylogenetic features as only $28.8 \%$ of genes were shared with its closest relative (van Passel et al. 2011). At present, whole-genome assembly of A. muciniphila can be sequenced directly from human stool in the absence of a cultured isolate (Caputo et al. 2015).

Akkermansia muciniphila plays an important role in maintaining a healthy mucus layer in the human gut (Derrien et al. 2017), and it may represent 3-5\% of the microbial composition in the healthy human intestinal tract (Derrien et al. 2004; Belzer and de Vos 2012). Studies have identified a loss in abundance of $A$. muciniphila in patients with obesity and type 2 diabetes (T2D) (Derrien et al. 2011; Everard et al. 2013). Moreover, increasing evidence has shown that the abundance of A. muciniphila is related with body weight, type 1 diabetes (T1D) (Hansen et al. 2012), inflammatory bowel disease (Png et al. 2010), autism (Wang et al. 2011) and cancer (Weir et al. 2013). The analysis of A. muciniphila genome predicts that over $61(11 \%)$ of proteins are involved in the degradation of mucin (Belzer and de Vos 2012). However, the precise physiological mechanisms affected by this bacterium during metabolic disorders and intestinal permeability regulation remain unclear, and much of the Akkermansia genus lacks in-depth analysis and comparative features among Akkermansia strains.
In the present study, 23 whole genome sequences of the Akkermansia genus were selected from NCBI databases for comparative studies. Digital DNA-DNA hybridization $(\mathrm{dDDH})$ values suggested that these whole genome sequences came from different species of the Akkermansia genus. Phylogenetic analysis of the concatenated singlecopy core genes suggested that 18 whole genome sequences formed a closely related clade. Pan-genome analyses were compared among these 18 genome sequences. Analysis of gene clusters and genomic islands suggested that these gene sequences were not conserved and that they may come from other species by lateral gene transfer (LGT).

\section{Materials and methods}

\section{Characterization of Akkermansia strains and closely related species}

Twenty-three strains of the Akkermansia genus were selected for comparative genome analysis from NCBI. The other 4 strains from the Verrucomicrobia phylum were selected based on species that were closely related to the Akkermansia genus. The genome sequences and annotation information for the strains were downloaded from NCBI databases.

\section{Genomic quality assessment}

The completeness and contamination of the genome sequences which recovered from metagenomes were evaluated by the software CheckM (Parks et al. 2015).

\section{dDDH values}

The results of the genome comparisons for taxonomic purpose were collected using BLAST2.7.1 + (Camacho et al. 2009). The digital DNA-DNA hybridization (dDDH) values were obtained by means of genome-to-genome sequence comparison via GGDC 2.0 using Formula 1 (Meier-Kolthoff et al. 2013), and the digital DNA-DNA hybridization $(\mathrm{dDDH})$ was used to replace the tedious traditional approach (Auch et al. 2010).

\section{Phylogenetic analysis}

Phylogenetic trees were constructed with the following three methods: two trees constructed using whole-genomebased methods; and the other tree was constructed using the concatenated single-copy core gene sequences. All amino acid sequences of each strain were used to construct the first phylogenetic tree using the web server of Composition Vector Tree Version 3 (CVTree3), and 6 was the K-tuple length (Zuo and Hao 2015). All dDDH values obtained 
from GGDC 2.0 were based on Genome-to-Genome Distances (GGDs) calculated by the Genome BLAST Distance Phylogeny (GBDP) approach (Henz et al. 2005; Auch et al. 2006), and the matrix of these GGDs was used to infer the second phylogenetic tree with FastME (Desper and Gascuel 2002). The concatenated single-copy core gene-based phylogenetic tree was generated through MEGA (Kumar et al. 2016) using the Neighbor Joining (NJ) method with 1000 bootstrap replicates, and Rubritalea marina DSM $18772^{\mathrm{T}}$ was the out-group. OrthoMCL was used to obtain orthologous genes of the strains to construct a phylogenetic tree $(\mathrm{Li}$ et al. 2003). The single-copy genes were obtained from the OrthoMCL results, and the concatenated single-copy core gene sequences were aligned using MAFFT (Katoh et al. 2017).

\section{Population structure}

Population structure was assessed by STRUCTURE 2.3.4 (Evanno et al. 2005) using the SNPs in core genes shared by 23 Akkermansia genomes. Of note, $K$ was varied from 1 to 8 with a burn-in of 5000 iterations. The best number of populations $(K)$ was identified using $\delta K$ via the method of Evanno et al. (2005).

\section{Variant calls: SNPs}

Firstly, single nucleotide polymorphism (SNP) calls were performed using Snippy, which uses BWA Mem (Li and Durbin 2009) to map the $250 \mathrm{bp}$ single-end reads that were shredded from the contigs to the reference A. muciniphila ATCC BAA- $835^{\mathrm{T}}$ and then calls the SNPs with FreeBayes (Garrison and Marth 2012). Whole genome alignment output from Snippy were used to identified the final result about SNPs distribution of the 18 A. muciniphila strains, using Gubbins (Genealogies Unbiased By recombinations In Nucleotide Sequences) (Croucher et al. 2015). The density of SNP distribution was calculated throughout the genome sequence using a sliding-window size of $5 \mathrm{~kb}$ (step of the sliding window $=5 \mathrm{~kb}$ ).

\section{Pan-genome analyses of Akkermansia muciniphila}

Roary is a high-speed pan genome pipeline (Page et al. 2015), which takes annotated assemblies in the GFF3 format and calculates the pan-genome. The GFF3 format files of the 18 genomes of $A$. muciniphila were produced using PROKKA (Seemann 2014). The gene accumulation curve was produced via ggplot2 using the results of Roary. A Venn diagram was used to show the core genes and strain-specifics of the 18 A. muciniphila strains.

\section{Identification of functional categories for core and strain-specific genes}

To identify the functional categories of core and strainspecific genes of the $18 \mathrm{~A}$. muciniphila strains, Clusters of Orthologous Groups (COGs) databases were used (Tatusov et al. 2003).

\section{Analyses of gene clusters and genomic islands}

The biosynthetic gene clusters of ATCC BAA- $835^{\mathrm{T}}$ strain were predicted using antiSMASH 4.0 (Blin et al. 2017). IslandViewer 4 was used to detect the genomic islands of ATCC BAA- $835^{\mathrm{T}}$ strain (Bertelli et al. 2017). The corresponding orthologous genes of the gene clusters and genomic islands in other strains were identified using OrthoMCL. All protein sequences were compared using BLASTP all-against-all with an E value cutoff of 1e-05 and 70\% similarity. The Markov Cluster (MCL) algorithm was used to determine orthologous groups using a value of 2.0 (Enright et al. 2002). The synteny maps of the gene clusters and genomic islands were generated using $\mathrm{R}$ package genoPlotR (Guy et al. 2011).

\section{Results}

\section{Genomic features and genomic quality assessment}

A summary of the features of each of the 23 genomes of the Akkermansia genus and 4 genomes of its closely related species is shown in Table 1 . The $\mathrm{G}+\mathrm{C}$ contents of the 23 genomes ranged from 55 to $58.1 \%$. Their genome sizes varied from 2.43 to $3.11 \mathrm{Mb}$ with the number of CDSs ranging from 2045 to 2526 . Compared with the other 4 genomes from the Verrucomicrobia phylum, the genomes of the Akkermansia genus were much smaller in size and had fewer CDSs, indicating the large difference between Akkermansia with other species of the Verrucomicrobia phylum. The genome sequences of the Akkermansia strains Urmite, CAG:154, CAG:344, UNK.MGS-1, 54_64, Phil8, UBA3271, UBA7059 and UBA7090 were recovered from metagenomes, so the genome may have sequence contamination. The software CheckM (Parks et al. 2015) was used to evaluate the genomes quality of these strains. The completeness and contamination of the sequences was listed in Table 1. The results suggested that these sequences have high quality with high sequence completeness and less contamination. 


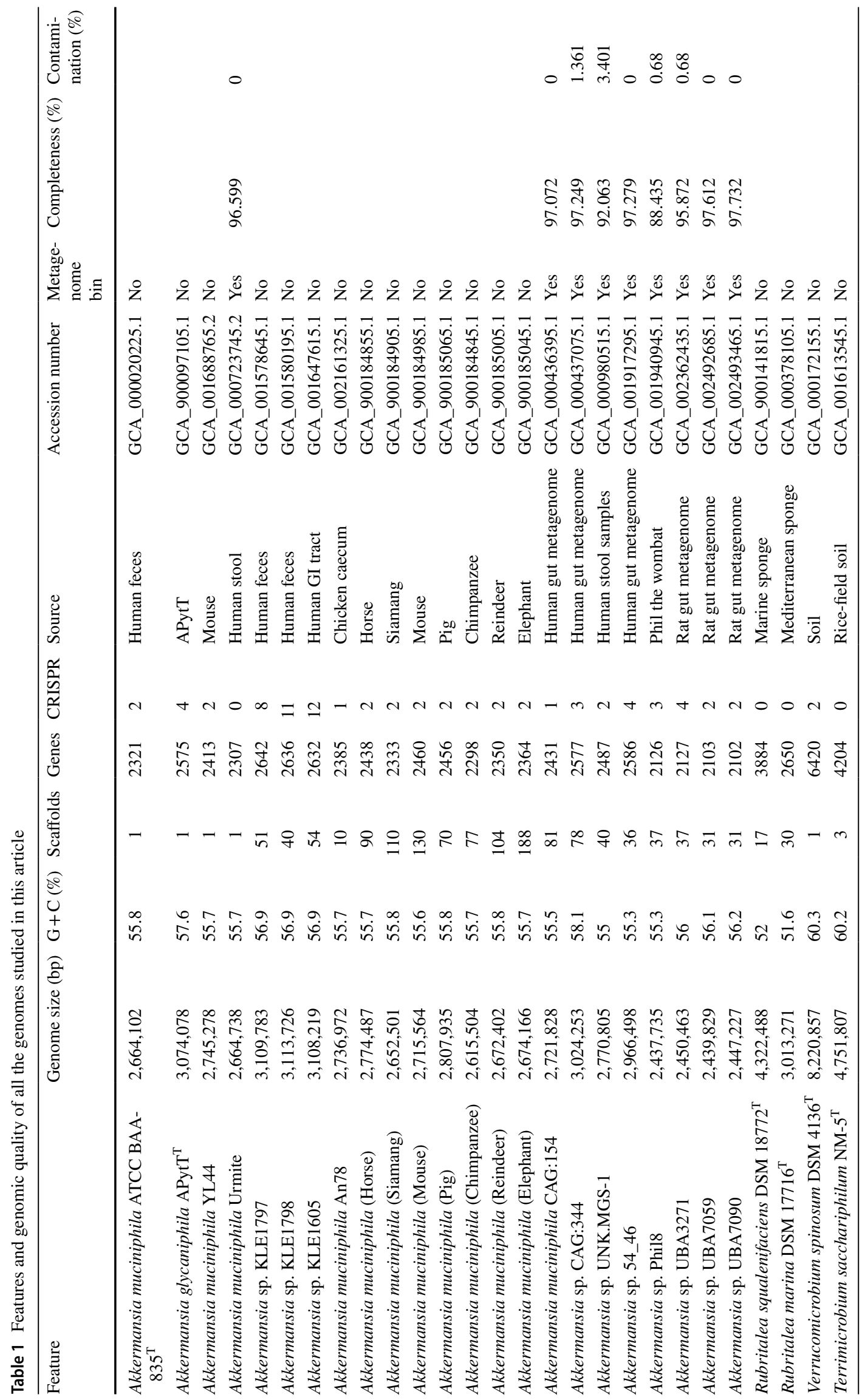




\section{dDDH values}

The matrix of digital DNA-DNA hybridization (dDDH) values of genome-to-genome is shown in Table S1. ANI values in the range of 95-96\% (Richter and Rossello-Mora 2009) correspond to a 70\% DDH standard for species definition (Wayne et al. 1987; Rosello-Mora and Amann 2001). Based on the species standard mentioned above, the 23 strains of the Akkermansia genus were divided into 4 species as follows: CAG:344 strain, a novel species of the Akkermansia genus; KLE1605 strain, KLE1797 strain and KLE1798 strain, which should be identified as another novel species of the Akkermansia genus. The two novel species were different from the published A. muciniphila and A. glycaniphila species, which was confirmed further by the following phylogenetic analysis.

\section{Phylogenetic analyses}

Two whole-genome-based methods were used to construct phylogenetic trees. Rubritalea marina DSM $17716^{\mathrm{T}}$, Rubritalea aqualenifaciens DSM $18772^{\mathrm{T}}$, Terrimicrobium sacchariphilum $\mathrm{NM}-5^{\mathrm{T}}$ and Verrucomicrobium spinosum DSM $4136^{\mathrm{T}}$ were selected as out-groups (Fig S1 and Fig S2) (Kasai et al. 2007; Scheuermayer et al. 2006; Qiu et al. 2014). The first method was alignment-free using whole amino acid sequences, and the second method depended on the distance matrix of all genome-to-genome comparisons (Table S2). The two phylogenetic trees were similar with 18 genome sequences of $A$. muciniphila clustered to one clade of the phylogenetic tree. The dDDH values of 17 other strains of this clade with A. muciniphila ATCC BAA$835^{\mathrm{T}}$ ranged from 75.5 to $99.9 \%$, which revealed that the 18 strains from the digestive tracts of human being, mouse, chimpanzee, elephant, horse, siamang, pig, reindeer, chicken and other animals can survive in diverse habitats, suggesting that Akkermansia has broad host adaptation. The genome sequences of $A$. muciniphila in different mammals were exactly similar with each other. The strains YL44 and Mouse isolated from mouse have close phylogenetic relation. And the strains UBA3271, UBA7090 and UBA7059 isolated from rat gut are in the same branch of the phylogenetic tree.

Concatenated core gene alignments are frequently used for constructing a genome-based phylogenetic tree. A total of 710 single-copy core genes were identified by comparison of 23 Akkermansia genomes and Rubritalea aqualenifaciens DSM $18772^{\mathrm{T}}$. The phylogenetic tree of the 23 Akkermansia genomes was constructed based on the concatenation of the 710 core genes that were present in single-copy in all genomes using the Neighbor-joining (NJ) method with 1000 bootstrap replicates (Fig. 1a) and rooted by Rubritalea aqualenifaciens DSM $18772^{\mathrm{T}}$. This phylogenetic tree was similar to the phylogenetic trees mentioned above, which further verified the phylogenetic status of the 23 strains of the Akkermansia genus.

\section{Population structure}

The population structure of the 23 Akkermansia genomes was investigated using STRUCTURE software based on the SNPs of core genes (Evanno et al. 2005). The result revealed that 23 Akkermansia strains were divided into 3 specific groups (Fig. 1b). The ATCC BAA-835, chimpanzee, elephant, horse, mouse, pig, reindeer, siamang, YL44, An78 and Urmite strains were clustered together, and most of genetic information of these strains was conserved. There was a little variation in the Urmite, UNK.MGS-1, 54_64, Phil8 and CAG: 154 , and strains ATCC BAA- $835^{\mathrm{T}}$, chimpanzee, elephant, horse, mouse, pig, reindeer, siamang, YL44 and An78 strains, showing high homology, which suggested that they evolved from a common ancestor. Phylogenetic analyses and dDDH values suggest that CAG:344 strain, KLE1605 and KLE1797 and KLE1798 should be classified as different species, and it'll be more rational that the Urmite, UNK.MGS-1, 54_64, Phil8 and CAG:154, ATCC BAA-835, chimpanzee, elephant, horse, mouse, pig, reindeer, siamang, YL44 and An78 strains come from a common ancestor through the population structure analyses method.

\section{Local diversification of poly-clade genomes}

To study the patterns of SNP distribution, SNP density was estimated throughout the 18 A. muciniphila genomes using a sliding window of $5 \mathrm{~kb}$. In total, 533 SNPs regions throughout the genomes and SNPs were not evenly distributed among these regions (Database S1). Of the 533 regions, 9 regions had low density SNPs (less than $2.0 \mathrm{SNPs} / \mathrm{kb}$ ), and 14 regions had high density SNPs (more than 75 SNPs/ $\mathrm{kb}$ ). The 9 regions with low density were related to Cell wall/membrane/envelope biogenesis. The 14 regions with high SNP density were related to metabolism and cell wall/ membrane envelope biogenesis.

\section{Pan-genome analysis of Akkermansia muciniphila}

To determine the core genes and strain-specific genes, 18 genomes of A. muciniphila were selected to analysis. Because some genomes were incomplete, a set of genes in their assemblies was missing. To determine the orthology genes, clustering was performed on the entire set of nucleotide sequences of all genes of each species instead of using sequence comparison against a reference strain. For the allagainst-all comparison of all sequences, $95 \%$ was defined as the percentage sequence identity standard of nucleotide sequence. 
Fig. 1 Phylogenetic relationship of 23 Akkermansia strains and population structure of 18 Akkermansia muciniphila strains. a Neighbor Joining (NJ) phylogenetic tree was constructed using the concatenated nucleotide sequences of 710 single-copy core genes shared by 23 Akkermansia genomes and an out-group (Rubritalea squalenifaciens DSM $18772^{\mathrm{T}}$ ). The phylogenetic tree was rooted by the out-group. b The 18 strains were divided into 3 populations $(K=3)$, and individuals are shown by thin vertical lines, which are divided into $K$ colored segments representing the estimated membership probabilities $(\mathrm{Q})$ of each individual (color figure online)

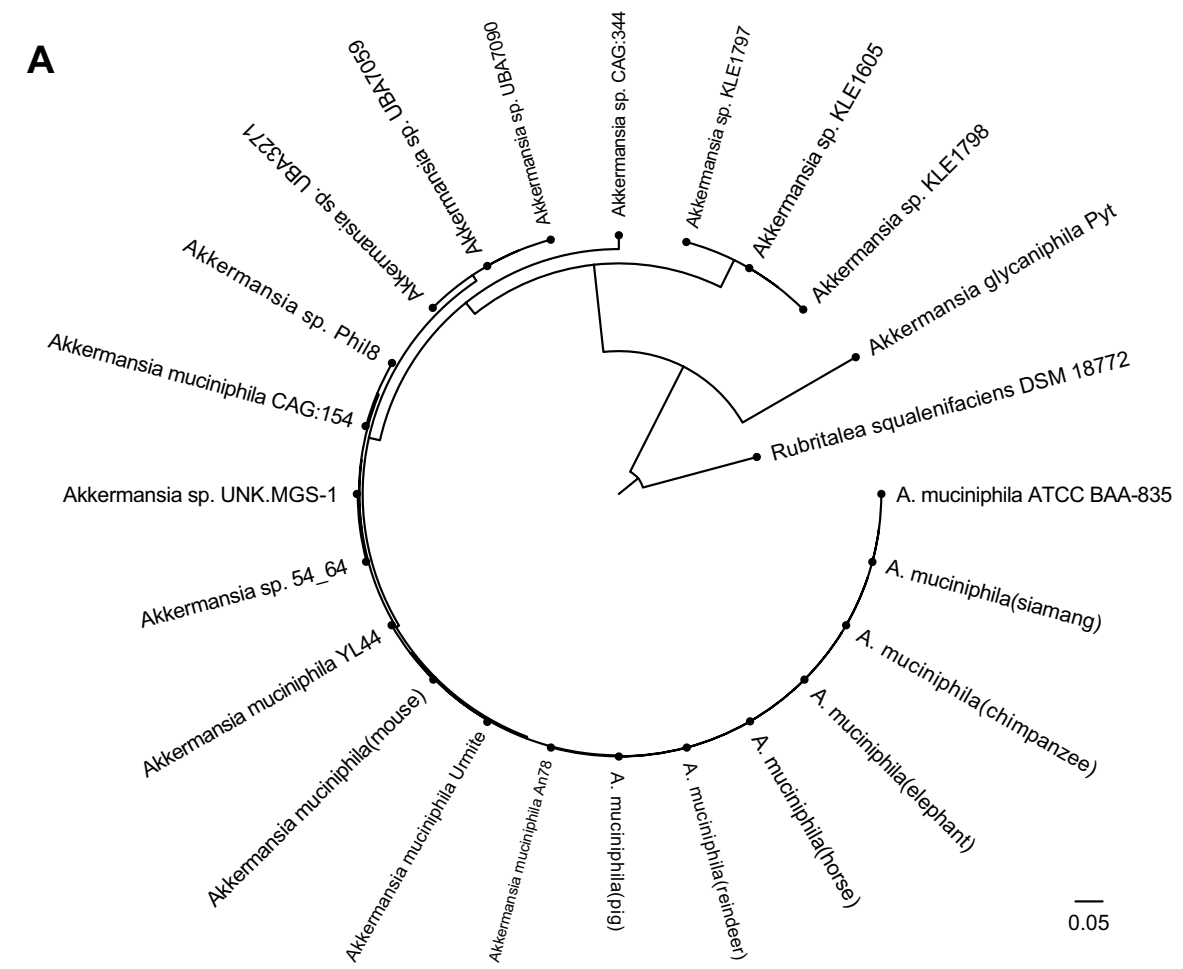

B

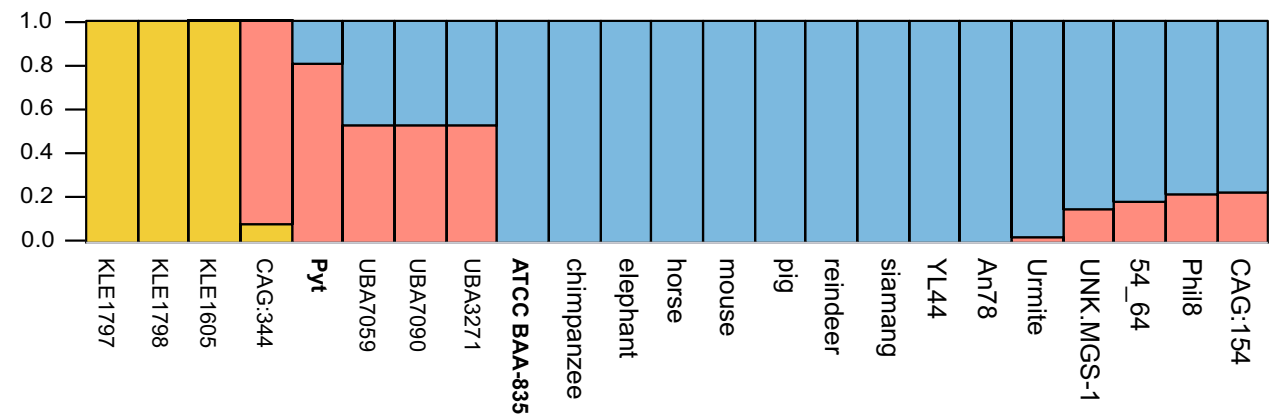

Among the 18 genomes, 5033 unique protein-coding genes were identified in the pan-genome, corresponding to more than two-fold the average genes of the 18 genomes. The gene accumulation curve showed that the numbers of the core genome genes decreased continually with the addition of new strains, while the pan-genome showed an increasing trend (Fig. 2a). The change of both curves slowed down because the pan-genome of Akkermanisa muciniphila was in an open state, indicating that unique genes would be added along with the addition of new strains. The gene occurrence plot showed that a core-genome containing 1035 genes was present in all $18 \mathrm{~A}$. muciniphila strains and that 1322 genes were strain-specific genes, and the plot also showed that the other genes were additional accessory genes mostly present in several genomes (Fig. 2b). The number of strain-specific genes in the 18 strains is shown in Fig. 2c. The remarkable difference of the specific genes between some strains suggested that these strains may have expanded in various habitats and that these strain-specific genes may have come from other species through lateral gene transfer (LGT). Furthermore, the strain-specific genes represented only a small number of strains, suggesting that the evolution of A. muciniphila was relatively conservative.

\section{Differentially enhanced functions in core and strain-specific genes}

After obtaining core and strain-specific gene sequences, the distribution of their functional categories was compared using the Clusters of Orthologous Groups (COGs) database (Tatusov et al. 2003). However, the functional categories of only $82.4 \%$ (852/1035) of core genes, $35.1 \%$ (464/1322) of strain-specific genes and 49.7\% (1331/2676) of accessory genes could be determined using the COGs database. Most of the genes did not exist in the COGs database, suggesting that $A$. muciniphila is poorly studied. The characteristics 

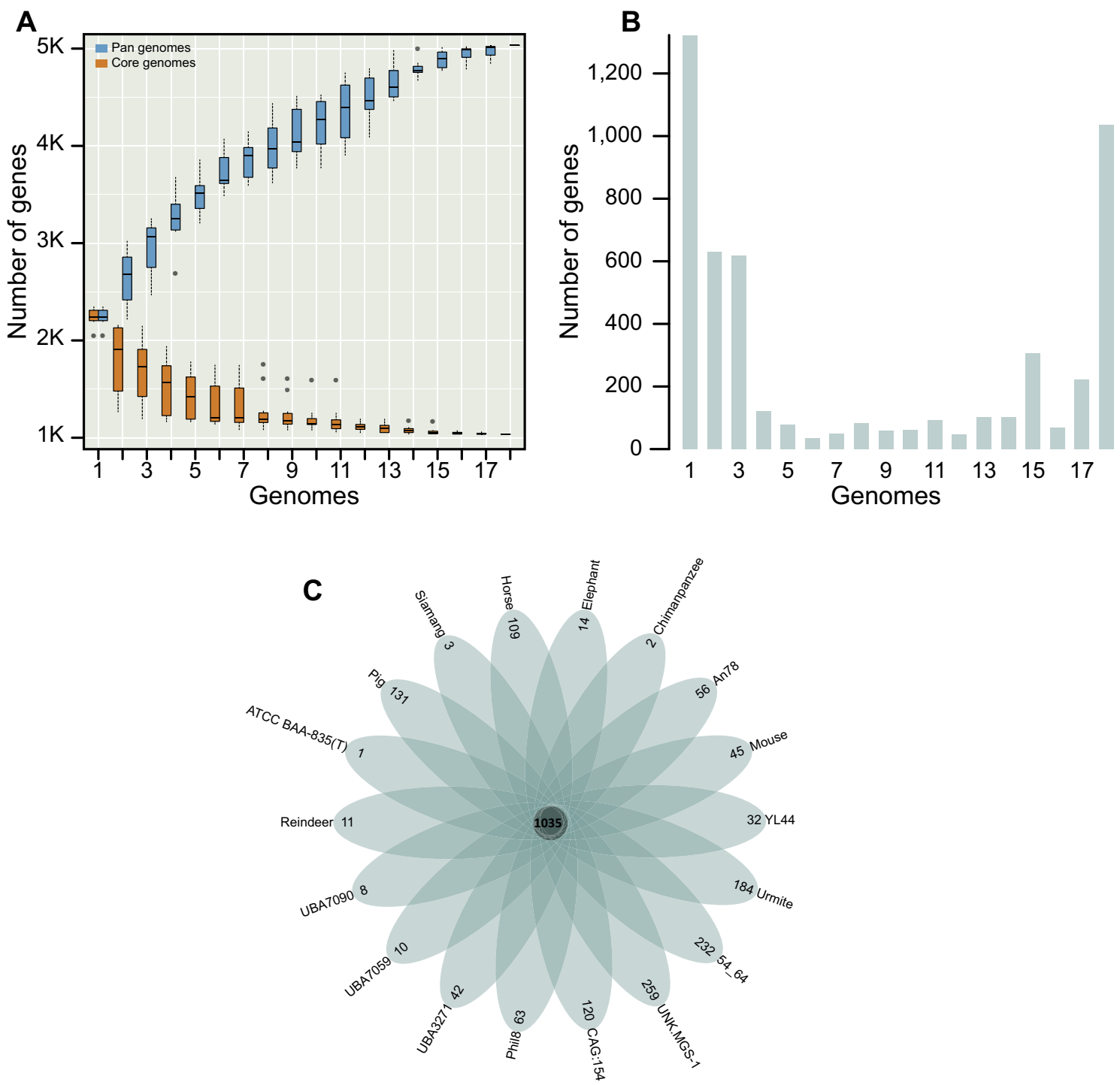

Fig. 2 Pan-genome analyses of 18 Akkermansia muciniphila strains. a Pan-genome accumulation curves. The blue boxes denote the number of unique genes discovered with the sequential addition of new genomes. The orange boxes denote the number of core genes discovered with the sequential addition of new genomes. b Gene occurrence plot shows the core-genome and additional accessory genes of Akker- mansia muciniphila. c Genomic diversity of 18 Akkermansia muciniphila strains. Each strain is shown as an oval. The number of core genomes is shown in the center. Overlapping regions show the genes conserved only within several strains. The numbers in non-overlapping portions show the number of strain-specific genes. The strain name is located beside the oval (color figure online) of relatively new species are scarce, thus suggesting the need to strengthen in-depth studies. This lack of in-depth knowledge may explain why functional categories could not be determined for many genes. The most abundant functions in the core genes of A. muciniphila were associated with metabolism (Fig. 3a). The overall proportion of genes related to metabolic functions was $39.6 \%, 30.4 \%$, and $33.4 \%$ in the core genes, strain-specific genes and accessory genes, respectively. More specifically, translation, ribosomal structure and biogenesis (J), amino acid transport and metabolism (E), and cell wall/membrane/envelope biogenesis (M) were abundant in the core genes, suggesting that these genes were relatively conserved in A. muciniphila. The mobilomerelated functions, such as prophage and transposase proteins $(\mathrm{X})$, were more abundant in strain-specific genes than core genes (1 Mobilome-related prophage and transposon $(\mathrm{X})$ in core genes; but 25 Mobilome-related prophage and transposon $(\mathrm{X})$ in strain-specific genes). These results suggested that these strain-specific genes may have been transferred horizontally from other species or even from another genus and that the functions were not relatively important (Fig. 3b).

The number of the specific genes among the 18 genomes was 1322 , ranging from 1 to 259 for each strain. The lowest number was encoded by A. muciniphila ATCC BAA- $835^{\mathrm{T}}$, 
A

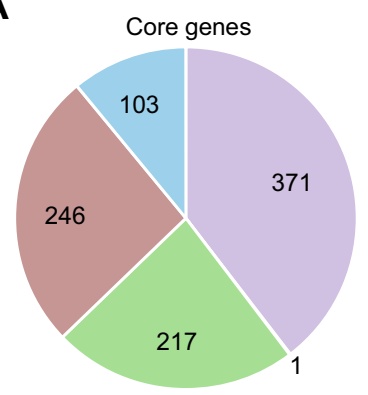

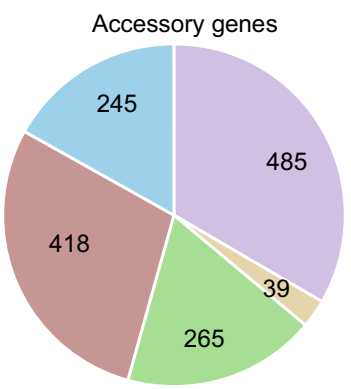

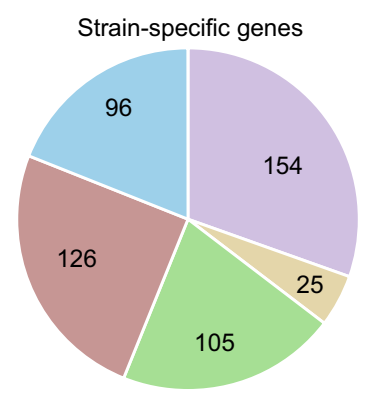

B

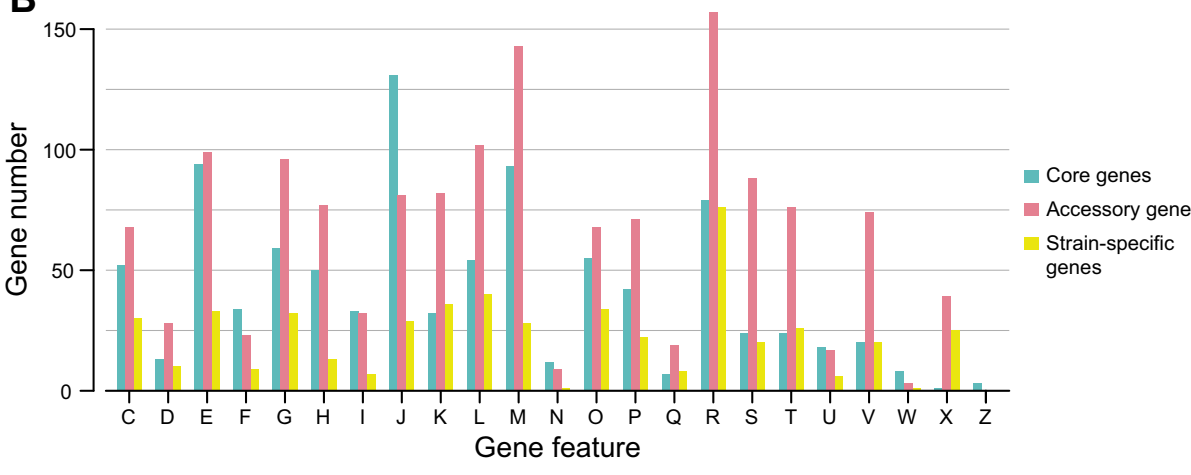

Metabolism

C Energy production and conversion

E Amino acid transport and metabolism

F Nucleotide transport and metabolism

G Carbohydrate transport and metabolism

H Coenzyme transport and metabolism

I Lipid transport and metabolism

$\mathrm{P}$ Inorganic ion transport and metabolism

Q Secondary metabolites biosynthesis, transport and catabolism

Mobileome

$X$ Mobilome: prophages, transposons

Information storage and processing

$\mathrm{J}$ Translation, ribosomal structure and biogenesis

$\mathrm{K}$ Transcription

$\mathrm{L}$ Replication, recombination and repair

Cellular processing and signaling

D Cell cycle control, cell division, chromosome partitioning

Cell wall/membrane/envelope biogenesis

$\mathrm{N}$ Cell motility

O Posttranslational modification,

protein turnover, chaperones

T Signal transduction mechanisms

$\cup$ Intracellular trafficking, secretion, and

vesicular transport

$\checkmark$ Defense mechanisms

W Extracellular structures

Z Cytoskeleton

Poorly characterized

$R$ General function prediction only

$S$ Function unknown

Fig. 3 Differential distribution of COG functional categories in core and strain-specific genes. a Proportion of five classes of functional categories in core, accessory and strain-specific genes. b Functional categories in core, accessory and strain-specific genes

and the highest number was identified in Akkermansia sp. UNK.MGS-1. Although a high number of strain-specific genes (approximately 65\%) was not assigned to the COG categories, the other strain-specific genes fell into different functional categories. A higher proportion of strain-specific genes in most of the strains was assigned to the transcription $(\mathrm{K})$, replication, recombination and repair $(\mathrm{L})$, carbohydrate transport and metabolism $(\mathrm{G})$, amino acid transport and metabolism (E), translation, ribosomal structure and biogenesis $(\mathrm{J})$ and energy production and conversion (C) categories (Fig S3).

\section{Analyses of gene clusters and genomic islands of Akkermansia}

The genomes of many microorganisms contain multiple biosynthetic gene clusters (BGCs) that code for production of secondary metabolites (Blin et al. 2017). These secondary metabolites play key roles in scientific research. The A. muciniphila ATCC BAA- $835^{\mathrm{T}}$ strain was selected as the reference to identify gene clusters using anti-SMASH 4.0 (Blin et al. 2017). Only 3 clusters responsible for the biosynthesis of secondary metabolites were identified as follows: 2 clusters belonged to terpenes; and 1 cluster belonged to arylpolyene. Moreover, the structures of the secondary metabolites coded by the 3 gene clusters could not be speculated.
The locations of the 3 gene clusters on the A. muciniphila ATCC BAA $-835^{\mathrm{T}}$ reference genome were from 421,014 to 441,913 , from $1,642,673$ to $1,683,848$ and from $1,745,606$ to $1,766,541$. The nucleotide sequence lengths of the 3 gene clusters were $20,899 \mathrm{bp}, 41,175 \mathrm{bp}$ and $20,935 \mathrm{bp}$, and the gene numbers of these clusters were 16, 26 and 25 , respectively. The secondary metabolites may play a part in prebiotic functions, and the isolation, purification and structure identification of these secondary metabolites should be completed in future studies.

Genomic islands (GIs) are a major driver of genome evolution as they often provide adaptive traits that enhance the fitness of bacteria and archaea within a niche (Aminov 2011; Dobrindt et al. 2004). Eight genomic islands were identified in the A. muciniphila ATCC BAA- $835^{\mathrm{T}}$ genome sequences, and the gene numbers of all genomic islands were 5, 6, 8, $12,7,23,5$ and 8 . The locations of these genomic islands are listed in Table S3. Compared with gene clusters, the genomic islands were smaller and included fewer genes. However, the functions of the genomic islands remain unknown. Analysis of all Akkermansia genomes revealed that only several strains had all 8 genomic islands mentioned above and that several strains only had a portion of the 8 genomic islands. These results suggested that the genes from genomic islands probably had a horizontal origin from other bacteria or archaea in different niches via different methods. 
Fig. 4 Comparison of biosynthetic gene clusters between Akkermansia muciniphila ATCC BAA- $835^{\mathrm{T}}$ and other strains of the Akkermansia genus. Regions of conserved synteny were marked with gray $(+)$ and green $(-)$ shadows. Different genes are shown by different color arrows, and genes with the same color are homologous to each other (color figure online)

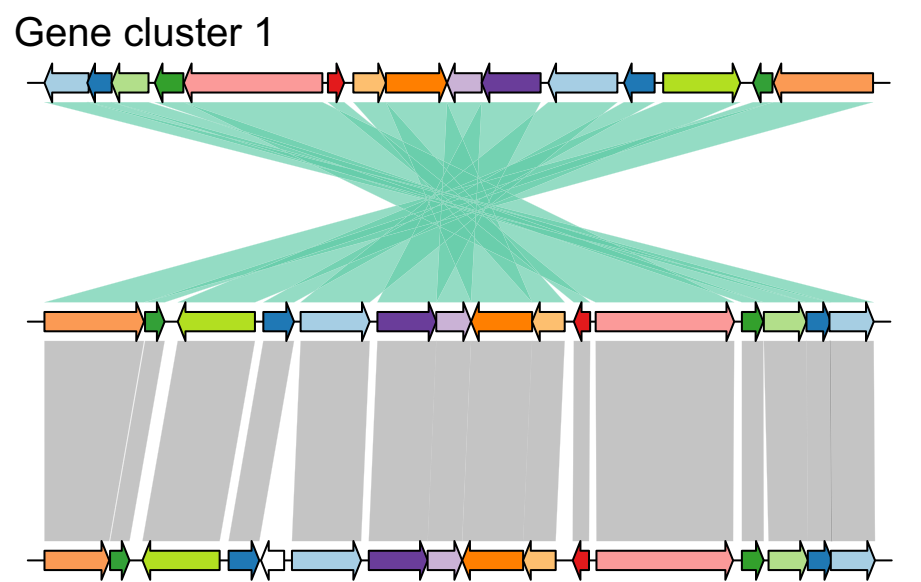

YL44

ATCC BAA-835(T)

CAG:344

$2 \mathrm{~kb}$

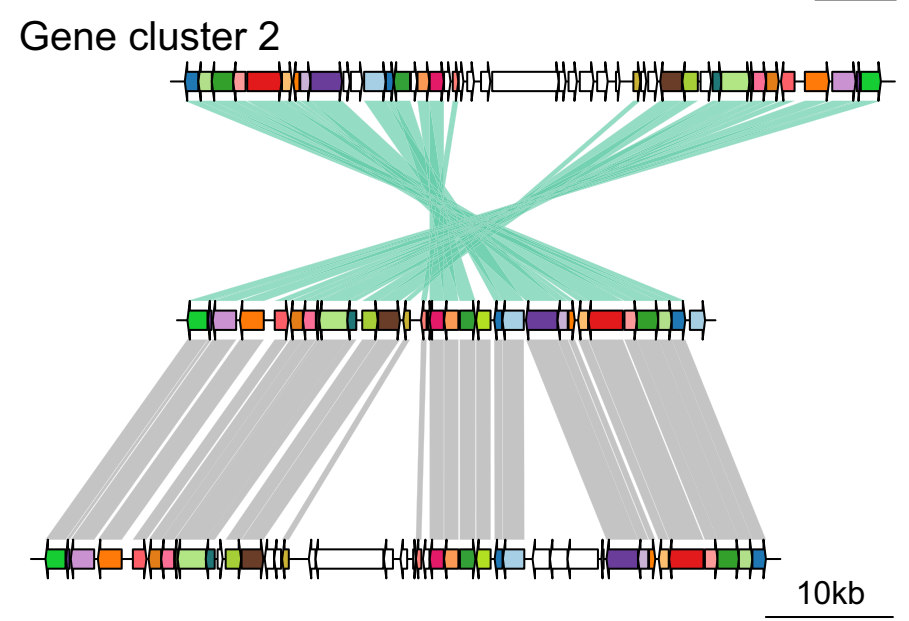

KLE1798

ATCC BAA-835(T)

CAG:344

\section{Gene cluster 3}

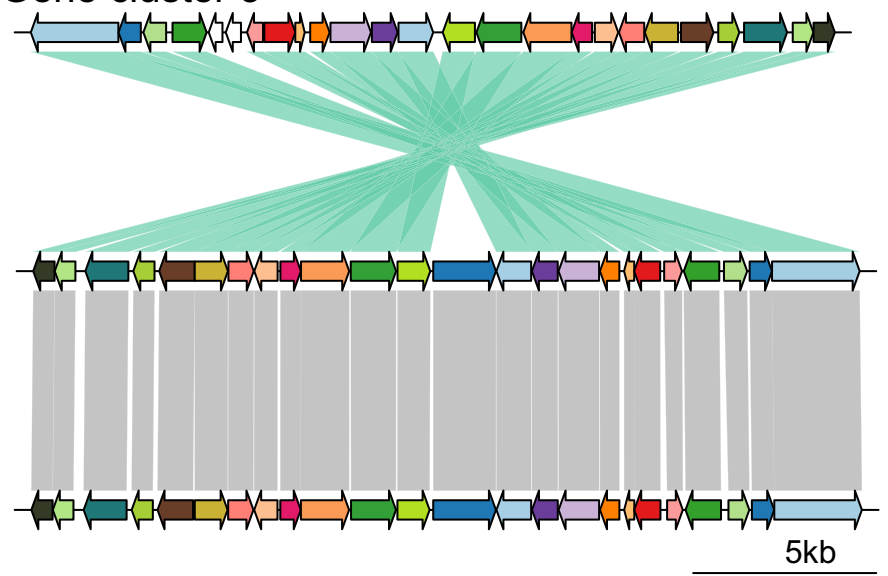

KLE1798

ATCC BAA-835(T)

CAG:344
Synteny analyses of the 3 gene clusters and the 8 genomic islands between $A$. muciniphila ATCC BAA- $835^{\mathrm{T}}$ and other strains of Akkermansia suggested that not all of the strains had these genes. The use of orthoMCL ( $\mathrm{Li}$ et al. 2003) determined that all orthologous genes of the 23 Akkermansia strains studied in this article had $70 \%$ sequence identity of standard amino acid sequences. A. muciniphila ATCC BAA $-835^{\mathrm{T}}$ was used as a reference to identify the same gene clusters and genomic islands in other strains. The $A$. glycaniphila $\mathrm{Pyt}^{\mathrm{T}}$ strain did not have similar gene clusters or genomic islands with the reference, which was potentially due to different evolution habitats. Furthermore, some clustered orthologous genes that were found in one strain had different positions, sequences, numbers, and orientation of the homologous genes among the strains, which may have been due to mutation, recombination and rearrangement events of these genes. The numbers and compositions of orthologous genes are listed in Table S4. The gene clusters 


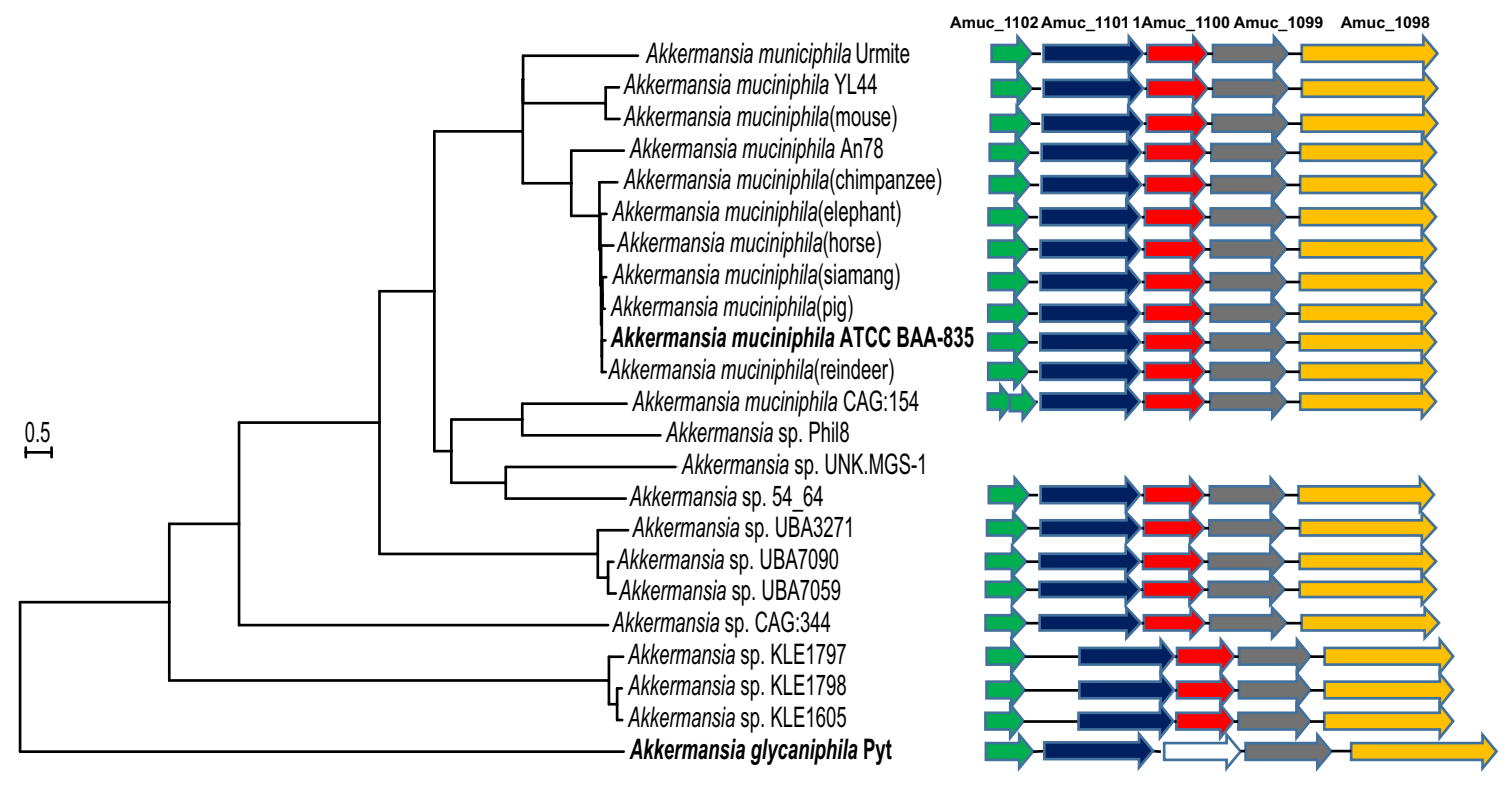

Fig. 5 A gene cluster corresponding to locus tags from Amuc_1098 to Amuc_1102 in 23 Akkermansia strains. The colors represent the following genes: green represents Amuc_1102, blue represents Amuc_1101, red represents Amuc_1100, gray represents Amuc_1099

were more conserved compared to genomic islands as shown in Table S5. Synteny maps comparing the gene clusters are shown in Fig. 4 and the genomic islands are shown in Figure S4.

\section{Analysis of Amuc_1100 cluster}

Amuc_1100, a thermostable outer-membrane protein of A. muciniphila has been reported to play an important role to reduce fat mass and metabolic syndrome in mice with diet-induced obesity (Anhê and Marette 2017). Locus tags for Amuc_1098 to Amuc_1102 were clustered into a gene cluster. Amuc_1098 was predicted to encode a type II and type III secretion system protein, and Amuc_1101 was predicted to encode a cell division protein FtsA. The other 3 genes were annotated as hypothetical proteins (Ottman et al. 2017). A. muciniphila, whether live or pasteurized, and Amuc_1100 could decreased high cholesterol levels. The function of Amuc_1100 was related to formation of pili, thus could participate in the interaction between the bacterium and Toll-like receptor2 (TLR2) (Cani and de Vos 2017). Because of the important function of Amuc_1100 and strong candidate for future drug development, so we make further analyses of this cluster. For this cluster, gene order, length and orientation were conserved throughout the Akkermansia genus (Fig. 5), for the CAG:154 strain genome. Amuc_1102 was divided into 2 genes, and this cluster was not present in the Phil8 and UNK.MGS-1 strains, which may have been due to incomplete genome drafts. The distance between Amuc_1101 and yellow represents Amuc_1098. The white arrow indicates that no homologous gene of Amuc_1100 existed in the Akkermansia glycaniphila $\mathrm{Pyt}^{\mathrm{T}}$ genome (color figure online)

and Amuc_1102 was longer for the KLE1605, KLE1797 and KLE1798 strains compared to the other strains. The homologous genes for the Amuc_1100 cluster of A. glycaniphila $\mathrm{Pyt}^{\mathrm{T}}$ were much longer. The homologous genes of Amuc_1100 in the Akkermansia genus were identified, except for the A. glycaniphila $\mathrm{Pyt}^{\mathrm{T}}$ strain, suggesting that A. glycaniphila $\mathrm{Pyt}^{\mathrm{T}}$ may not have the similarity function as other species in the Akkermansia genus.

\section{Genes related to mucin degradation}

The analysis of A. muciniphila ATCC BAA- $835^{\mathrm{T}}$ genome predicts that over $61(11 \%)$ of proteins are involved in the degradation of mucin (Belzer and de Vos 2012). Predicted function of these genes has been mentioned in the previous study performed by other groups (Derrien et al. 2010). Comparative analysis of the other strains and $A$. muciniphila ATCC BAA- $835^{\mathrm{T}}$, the distributions of these genes in different strains were displayed in Table S5. The results suggested that the strains which were more related to A. muciniphila ATCC BAA- $835^{\mathrm{T}}$ own more homologous genes related to mucin degradation.

\section{Discussion}

Phylogenetic and DDH similarity analyses of 23 strains of the Akkermansia genus indicated that there are 4 species in this genus besides the 2 previously published 
Akkermansia species. Despite various habitats, the $A$. muciniphila strains were similar, suggesting that the evolution of A. muciniphila was relatively conservative. Population structure analysis supported the phylogenetic analysis results. Pan-genome analysis of 18 A. muciniphila genome sequences showed that the strain-specific genes were only a small proportion for each strain. The gene accumulation curve indicated that the pan-genome of $A$. muciniphila was in the open state and that it increased with the addition of new strains. Synteny analyses of gene clusters and genomic islands showed that only several strains had homologous sequences with A. muciniphila ATCC BAA- $835^{\mathrm{T}}$. The important thermostable outer-membrane protein, Amuc_1100, which is related to fat mass and metabolic syndrome, was not present in Akkermasia glycaniphila $\mathrm{Pyt}^{\mathrm{T}}$. Therefore, the characteristics of the important gut probiotic, Akkermasia, remain unknown, and future efforts need to be developed for in-depth understanding and possible application of the probiotic.

Acknowledgements This work was supported by the National Key Research and Development Program of China (2017YFD0201401) and National Natural Science Foundation of China (NSFC No.31670113 and NSFC No.31670011).

\section{Compliance with ethical standards}

Conflict of interest The authors declare no competing interests.

Open Access This article is distributed under the terms of the Creative Commons Attribution 4.0 International License (http://creativeco mmons.org/licenses/by/4.0/), which permits unrestricted use, distribution, and reproduction in any medium, provided you give appropriate credit to the original author(s) and the source, provide a link to the Creative Commons license, and indicate if changes were made.

\section{References}

Aminov RI (2011) Horizontal gene exchange in environmental microbiota. Front Microbiol 2:158

Anhê FF, Marette A (2017) A microbial protein that alleviated metabolic syndrome. Nat Med 23:11-12

Auch AF, Henz SR, Holland BR, Göker M (2006) Genome BLAST distance phylogenies inferred from whole plastid and whole mitochondrion genome sequences. BMC Bioinform 7:350

Auch AF, von Jan M, Klenk HP, Göker M (2010) Digital DNADNA hybridization for microbial species delineation by means of genome-to-genome sequence comparison. Stand Genom Sci 2:117-134

Belzer C, de Vos WM (2012) Microbes inside-from diversity to function: the case of Akkermansia. ISME J 6:1449-1458

Bertelli C, Laird MR, Williams KP, Simon Fraser University Research Computing Group (2017) IslandViewer 4: expanded prediction of genomic islands for larger-scale datasets. Nucl Acids Res 45:W30-W35

Blin K, Wolf T, Chevrette MG, Lu X (2017) AntiSMASH 4.0-improvements in chemistry prediction and gene cluster boundary identification. Nucl Acids Res 45:W36-W41
Camacho C, Coulouris G, Avagyan V, Ma N (2009) BLAST + : architecture and applications. BMC Bioinform 10:421

Cani PD, de Vos VM (2017) Next-generation beneficial microbes: the case of Akkermansia muciniphila. Front Microbiol 8:1765

Caputo A, Dubourg G, Croce O, Gupta S (2015) Whole-genome assembly of Akkermansia muciniphila sequenced directly from human stool. Biol Direct 10:5

Costello EK, Gordon JI, Secor SM, Knight R (2010) Postprandial remodeling of the gut microbiota in Burmese pythons. ISME $\mathrm{J}$ 4:1375-1385

Croucher NJ, Page AJ, Connor TR, Delaney AJ (2015) Rapid phylogenetic analysis of large samples of recombinant bacterial whole genome sequences using Gubbins. Nucl Acids Res 43:e15

Derrien M, Vaughan EE, Plugge CM, Vos WM (2004) Akkermansia muciniphila gen. nov., sp. nov, a human intestinal mucin-degrading bacterium. Int J Syst Evol Microbiol 54:1469-1476

Derrien M, van Passel MWJ, van de Bovenkamp JHB, Schipper RG (2010) Mucin-bacterial interactions in the human oral cavity and digestive tract. Gut Microbe 1(4):254-268

Derrien M, Baarlen PV, Hooiveld G, Norin E (2011) Modulation of mucosal immune response, tolerance, and proliferation in mice colonized by the mucin-degrader Akkermansia muciniphila. Front Microbiol 2:166

Derrien M, Belzer C, de Vos WM (2017) Akkermansia muciniphila and its role in regulating host functions. Microb Pathog 106:171-181

Desper R, Gascuel O (2002) Fast and accurate phylogeny reconstruction algorithms based on the minimum-evolution principle. $\mathrm{J}$ Comput Biol 9:687-705

Dobrindt U, Hochhut B, Hentschel U, Hacker J (2004) Genomic islands in pathogenic and environmental microorganisms. Nat Rev Microbiol 2:414-424

Enright AJ, Van Dongen S, Ouzounis CA (2002) An efficient algorithm for large-scale detection of protein families. Nucl Acids Res 30:1575-1584

Evanno G, Regnaut S, Goudet J (2005) Detecting the number of clusters of individuals using the sofware STRUCTURE: a simulation study. Mol Ecol 14:2611-2620

Everard A, Belzer C, Geurts L, Quwerkerk JP (2013) Cross-talk between Akkermansia muciniphila and intestinal epithelium controls diet-induced obesity. Proc Natl Acad Sci USA 110:9066-9071

Garrison E, Marth G (2012) Haplotype-based variant detection from short-read sequencing. arXiv preprint arXiv: 1207.3907 [q-bio. $\mathrm{GN}]$

Guy L, Kultima JR, Andersson SG (2011) genoPlotR: comparative gene and genome visualization in R. Bioinformatics 27:2334-2335

Hansen CH, Krych L, Nielsen DS, Vogensen FK (2012) Early life treatment with vancomycin propagates Akkermansia muciniphila and reduces diabetes incidence in the NOD mouse. Diabetologia 55:2285-2294

Hedlund BP, Gosink JJ, Staley JT (1997) Verrucomicrobia div. nov., a new division of the Bacteria containing three new species of Prosthecobacter. Antonie Van Leeuwenhoek 72:29-38

Henz SR, Huson DH, Auch AF, Nieselt-Struwe K (2005) Wholegenome prokaryotic phylogeny. Bioinformatics 21:2329-2335

Kasai H, Katsuta A, Sekjguchi H, Matsuda S (2007) Rubritalea squalenifaciens $\mathrm{sp}$. nov., a squalene-producing marine bacterium belonging to subdivision 1 of the phylum 'Verrucomicrobia'. Int J Syst Evol Microbiol 54:1630-1634

Katoh K, Misawa K, Kuma K, Miyata T (2002) MAFFT: a novel method for rapid multiple sequence alignment based on fast Fourier transform. Nucleic Acids Res 30:3059-3066

Kumar S, Stecher G, Tamura K (2016) MEGA7: molecular evolutionary genetics analysis version 7.0 for bigger datasets. Mol Biol Evol 33:1870-1874 
Ley R, Hamady M, Lozupone C, Turnbaugh PJ (2008) Evolution of mammals and their gut microbes. Science 320:1647-1651

Li H, Durbin R (2009) Fast and accurate short read alignment with Burrows-Wheeler transform. Bioinformatics 25:1754-1760

Li L, Stoeckert CJ Jr, Roos DS (2003) OrthoMCL: identification of ortholog groups for eukaryotic genomes. Genome Res 13:2178-2189

Meier-Kolthoff JP, Auch AF, Klenk HP, Göker M (2013) Genome sequence-based species delimitation with confidence intervals and improved distance functions. BMC Bioinform 14:60

Ottman N, Reunanen J, Meijerink M, Pietilä TE (2017) Pili-like proteins of Akkermansia muciniphila modulate host immune responses and gut barrier function. PLoS One 12:e0173004

Ouwerkerk JP, Aalvink S, Belzer C, Vos WM (2016) Akkermansia glycaniphila sp. nov., an anaerobic mucin-degrading bacterium isolated from reticulated python faeces. Int J Syst Evol Microbiol 66:4614-4620

Ouwerkerk JP, Koehorst JJ, Schaap PJ, Ritari J (2017) Complete genome sequence of Akkermansia glycaniphila strain $\mathrm{Pyt}^{\mathrm{T}}$, a mucin-degrading specialist of the reticulated python gut. Genome Announc 5:e01098-16

Page AJ, Cummins CA, Hunt M, Wong VK (2015) Roary: rapid large-scale prokaryote pan genome analysis. Bioinformatics 31:3691-3693

Parks DH, Imelfort M, Skennerton CT, Hugenholtz P (2015) CheckM: assessing the quality of microbial genomes recovered from isolates, single cells, and metagenomes. Genome Res 25:1043-1055

Png CW, Lindén SK, Gilshenan KS, Zoetendal EG (2010) Mucolytic bacteria with increased prevalence in IBD mucosa augment in vitro utilization of mucin by other bacteria. Am J Gastroenterol 105:2420-2428

Presley LL, Wei B, Braun J, Borneman J (2010) Bacteria associated with immunoregulatory cells in mice. Appl Environ Microbiol 76:936-941

Qiu YL, Kuang XZ, Shi XS, Yuan XZ (2014) Terrimicrobium sacchariphilum gen. nov., sp. nov., an anaerobic bacterium of the class 'Spartobacteria' in the phylum Verrucomicrobia, isolated from a rice paddy field. Int J Syst Evol Microbiol 64:1718-1723
Richter M, Rossello-Mora R (2009) Shifting the genomic gold standard for the prokaryotic species definition. Proc Natl Acad Sci USA 106:19126-19131

Roeselers G, Mittge EK, Stephens WZ, Parichy DM (2011) Evidence for a core gut microbiota in the zebrafish. ISME J 5:1595-1608

Rosello-Mora R, Amann R (2001) The species concept for prokaryotic. FEMS Microbiol Rev 25:39-67

Scheuermayer M, Gulder TA, Bringmann G (2006) Rubritalea marina gen. nov., sp. nov., a marine representative of the phylum 'Verrucomicrobia', isolated from a sponge (Porifer). Int J Syst Evol Microbiol 56:2119-2124

Seemann T (2014) Prokka: rapid prokaryotic genome annotation. Bioinformatics 30:2068-2069

Tatusov RL, Fedorrova ND, Jackson JD, Jacobs AR (2003) The COG database: an updated version includes eukaryotes. BMC Bioinform 4:41

van Passel M, Kant R, Zoetendal EG, Plugge CM (2011) The genome of Akkermansia muciniphila, a dedicated intestinal mucin degrader, and its use in exploring intestinal metagenomes. PLoS One 6:e16876

Wang L, Christophersen CT, Sorich MJ, Gerber JP (2011) Low relative abundances of the mucolytic bacterium Akkermansia muciniphila and Bifidobacterium spp. in feces of children with autism. Appl Environ Microbiol 77:6718-6721

Wayne LG, Brenner DJ, Colwell RR, Grimont PAD (1987) Report of the ad hoc committee on reconciliation of approaches to bacterial systematics. Int J Syst Bacteriol 37:463-464

Weir TL, Manter DK, Sheflin AM, Barnett BA (2013) Stool microbiome and metabolome differences between colorectal cancer patients and healthy adults. PLoS One 8:e70803

Zuo G, Hao B (2015) CVTree3 web server for whole-genome-based and alignment-free prokaryotic phylogeny and taxonomy. Genom Proteom Bioinform 13:321-331

Publisher's Note Springer Nature remains neutral with regard to jurisdictional claims in published maps and institutional affiliations.

\section{Affiliations}

\section{Juyuan Xing ${ }^{2}$. Xiaobo $\mathrm{Li}^{3,5,6}$. Yingjiao Sun ${ }^{1}$ Juanjuan Zhao ${ }^{1}$. Shaohua Miao ${ }^{1}$ Q Qin Xiong ${ }^{1}$. Yonggang Zhang ${ }^{4}$. Guishan Zhang'}

Juyuan Xing

junru1120102514@gmail.com

Xiaobo Li

815752398@qq.com

Yingjiao Sun

1153501575@qq.com

Juanjuan Zhao

zhaojuanjuan3867@163.com

Shaohua Miao

1536115780@qq.com

Qin Xiong

skyxiongqin@163.com

1 Key Laboratory of Microbial Resources Collection and Preservation, Ministry of Agriculture, Institute of Agricultural Resources and Regional Planning, Chinese Academy of Agricultural Sciences, No. 12 Zhongguancun
South Street, Haidian District, Beijing 100081, People's Republic of China

2 Wuhan University of Technology, Wuhan, Hubei, People's Republic of China

3 BGI Education Center, University of Chinese Academy of Sciences, Shenzhen, China

4 Biology Institute, Qilu University of Technology (Shandong Academy of Sciences), No. 19 Keyuan Road, Jinan 250014, Shandong, People's Republic of China

5 BGI-Shenzhen, Shenzhen, China

6 China National GeneBank-Shenzhen, BGI-Shenzhen, Shenzhen, China 